\title{
Governança do Conhecimento (GovC): o estado da arte sobre o termo
}

\author{
Patricia de Sá Freire \\ Gertrudes Aparecida Dandolini \\ João Artur de Souza \\ Talita Caetano Silva \\ Rogéria Moreira Couto \\ Universidade Federal de Santa Catarina - UFSC, Brasil
}

O R IG IN A L

\begin{abstract}
Resumo
Objetivo. No contexto organizacional contemporâneo o compartilhamento e a transferência do conhecimento têm um papel significativo e, por isso, é importante superar as barreiras internas e externas para que estes sejam processados. O que pode ser facilitado com a implantação da Governança do Conhecimento (GovC), uma abordagem interdisciplinar emergente, que atravessa os campos da gestão do conhecimento, os estudos de organização, estratégia e gestão de recursos humanos. O problema é que, além de ser um construto novo e ainda pouco estudado no Brasil, alimentam-se divergências conceituais pela amplitude de dimensões de análise possíveis. Neste contexto, surge o objetivo deste estudo, que seja, identificar a conceituação do construto Governança do Conhecimento proposta na literatura científica para apoiar sua melhor compreensão e embasar futuras pesquisas na área de gestão do conhecimento.

Método. Para tal, foi realizada uma pesquisa teórica por meio de uma sistemática revisão da literatura, seguida de análise bibliométrica e descritiva das publicações mais relevantes sobre o tema.

Resultados. O objetivo foi alcançado sendo possível identificar a conceituação, além dos princípios e dos mecanismos de GovC propostos pela literatura.

Conclusões. Considera-se a discussão sobre o constructo GovC pertinente no contexto das organizações contemporâneas, no entanto, sinaliza-se a importância de estudos futuros de ordem empírica e teórica de modo a fomentar as discussões sobre o assunto na atualidade
\end{abstract}

Palavras-chave

Conhecimento; Gestão do Conhecimento; Gorvenança Corporativa; Governança do Conhecimento

\section{Knowledge Governance (GovC): The State of the Art about the Term}

Abstract

Objective. In the contemporary organizational context sharing and knowledge transfer play a significant role and it is therefore important to overcome the internal and external barriers for processing. This can be facilitated by the implementation of Knowledge Governance (GovC), an emerging interdisciplinary approach that goes through the fields of knowledge management, organizational studies, strategy and human resources management. The problem is that, besides being a new construct and still little studied in Brazil, conceptual differences are fed by the breadth of possible dimensions of analysis. In this context, the objective of this study is to identify the conceptualization of the Knowledge Governance construct proposed in the scientific literature to support its better understanding and to base future research in the area of knowledge management

Method. For this, a theoretical research was carried out through a systematic review of the literature, followed by bibliometric and descriptive analysis of the most relevant publications on the subject.

Results. The objective was achieved and it is possible to identify the conceptualization, besides the principles and mechanisms of GovC proposed in the literature.

Conclusions. It is considered the discussion about the relevant GovC construct in the context of contemporary organizations, however, it indicates the importance of future empirical and theoretical studies in order to foment the discussions on the subject nowadays. 


\section{Introdução}

O constructo Gestão do Conhecimento tem despertado a atenção dos estudos acadêmicos desde a década de 60. O primeiro artigo publicado sobre o tema, registrado na base internacional de dados Scopus data de 1966 (BYRD et al., 1966). Nos últimos anos, precisamente após a década de 80 , houve um aumento na produção científica em busca de sua conceituação e da delimitação de seus processos, tratando o conhecimento como um importante recurso organizacional.

$\mathrm{Na}$ atual economia do conhecimento, para vencer os desafios do mundo sem fronteiras e da contemporânea desglobalização, é imprescindível a gestão dos conhecimentos essenciais para agregar valor distintivo às organizações. Assim, alguns modelos organizacionais demandam criticamente a implantação da gestão do conhecimento, pois estes levam em consideração o conhecimento como o principal ativo. Entre os modelos destacam-se os modelos de Frame Break (MITROFF et al, 1994); Organização inovadora (GALBRAITH, 1997); Organização em hipertexto (NONAKA E TAKEUCHI, 1997); Capital Intelectual (SVEIBY, 1998); Organização individualizada (GOSHAL E BARTLET, 2000) e o Modelo de Cadeia de Conhecimento (HOLSAPPLE E SINGH, 2001).

Para todos os modelos citados (MITROFF ET AL, 1994; GALBRAITH, 1997; NONAKA e TAKEUCHI, 1997; GOSHAL e BARTLET, 2000; HOLSAPPLE e SINGH, 2001), o desenvolvimento organizacional surge a partir das práticas de gestão do conhecimento e podem se manifestar pela criatividade dos funcionários; pela flexibilidade com que a organização reage ao ambiente; pela produtividade que alcança e, sobretudo, pela capacidade de inovação que possui. Ou seja, todas seriam dependentes da governança de estruturas, sistemas e políticas potencializadoras da gestão do conhecimento para que seja possível atingir os resultados pretendidos.

Como afirmam Alves e Barbosa (2010), tanto no contexto externo que exige a participação das organizações públicas e privadas em redes de aprendizagem, como no ambiente intra organizacional que demanda diálogos interdepartamentais mais eficazes, o processo de compartilhamento do conhecimento tem um papel significativo e, por isso, é importante superar as barreiras internas e externas para sua efetividade. Neste sentido, a implantação de uma governança corporativa que reconheça o valor do "conhecimento" nos processos de negócios pode favorecer tanto a aprendizagem organizacional como a aprendizagem em rede.

Porém, ainda é nova a discussão sobre princípios e mecanismos de governança que deverão nortear um modelo de governança do conhecimento para que apoie a gestão do conhecimento e, consequentemente, o alcance dos objetivos estratégicos da organização, visto que o termo "Governança do Conhecimento" só foi cunhado ao final da década de 90 pela pesquisadora Anna Grandori (1997) em seu artigo "Knowledge governance". E, mesmo com a passagem em torno de vinte anos, pouco se publicou sobre esta dimensão de análise do conhecimento organizacional, tendo a academia pesquisado mais sobre os processos de gestão de conhecimento ao invés dos mecanismos de governança para que estes fossem avaliados, controlados e monitorados. O que levou à escassez de literatura sobre o termo principalmente na língua portuguesa. Foram mapeadas apenas 35 publicações em base de dados internacional e uma no Brasil, no banco de teses de dissertações da Capes.

Entre os que veem pesquisando sobre governança do conhecimento, nos primórdios dos anos 2000, surgem os estudos de Bart Nooteboom da Rotterdam School of Management da Erasmus University, contextualizando a importância da Governança dos conhecimentos criados nos relacionamentos interorganizacionais. Já em 2003, o pesquisador Nicolai J. Foss e seus colaboradores do Centro de Gestão Estratégica e Globalização da Escola de Negócios de Copenhagen explicam que, teoricamente, não havia aquele tempo uma teoria disciplinar que explicasse a governança do conhecimento (GovC). Ao aprofundar suas pesquisas interdisciplinares, precisamente em 2007, Foss traz a ideia de Knowledge Governance Approach (KGA), onde define GovC como uma abordagem interdisciplinar emergente, que atravessa os campos da gestão do conhecimento, dos estudos de organização, da estratégia e da gestão de recursos humanos.

Em 2008, amplia-se o debate sobre a conceituação de Governança do Conhecimento quando surgem escolas europeia referindo-se ao construto como Knowledge Governance ou como Governance of Knowledge. 
Parece lógico que, alimentam-se divergências conceituais sobre o constructo Governança (SANTISO,2001) pela diversidade de sua aplicação (global, mundial, corporativa, entre outras) e a amplitude de dimensões de análise possíveis da própria gestão do conhecimento organizacional, o que gera a falta de clareza sobre o construto Governança do Conhecimento. Neste contexto, surge o objetivo deste estudo, que seja, identificar a conceituação do construto Governança do Conhecimento proposta na literatura científica para apoiar sua melhor compreensão e embasar futuras pesquisas na área de gestão do conhecimento organizacional. Para tal, foi realizada uma pesquisa teórica por meio de uma sistemática revisão da literatura, seguida de análise bibliométrica e descritiva das publicações mais relevantes sobre o tema.

O artigo é apresentado em cinco seções, a primeira sendo esta introdução, seguida da apresentação dos procedimentos metodológicos e dos resultados quantitativos da revisão sistemática da literatura. A terceira e a quarta seção apresentam respectivamente as análises bibliométrica e descritiva sobre GovC. Por último, são apresentadas as conclusões e finalmente, as referências utilizadas para este estudo.

\section{Procedimentos metodológicos}

Esta pesquisa pode ser classificada como descritiva com etapa exploratória por meio bibliográfico (MARCONI; LAKATOS, 2009) utilizando-se dos sete passos da revisão sistemática da literatura determinado pelo Cochrane Handbook, pois objetiva clarificar conceitos com base em publicações científicas qualificadas para delinear o tema abordado. Como uma pesquisa teórica, realizou-se a identificação e descrição dos estudos já realizados sobre GovC, promove-se um diálogo subjetivo entre diferentes autores (FREIRE, 2013).

A partir das análises - bibliométrica e descritiva pôde-se realizar a síntese do conhecimento sobre GovC levando a uma melhor precisão na avaliação dos conceitos e propostas dos diferentes autores.

No que diz respeito ao planejamento da revisão foi elaborado o protocolo da pesquisa bibliográfica, definindo a pergunta da revisão, os critérios de inclusão e exclusão e, as estratégias de buscas com a definição de onde se queria chegar e os critérios para a avaliação crítica. Assim, definiu-se como pergunta da presente revisão: qual o tamanho, crescimento e distribuição da bibliografia sobre Governança do Conhecimento?

Com a intenção de realizar a captura de publicações que tratam dos termos "Governança do Conhecimento" e/ou, em inglês, "Governance of Knowledge' e/ou "Knowledge Governance", estas três expressões foram consideradas descritores das buscas. Assim, como critério de inclusão, foram consideradas as publicações que se referiam aos termos selecionados no título, nos resumos e nas palavras chave. Pontua-se que nenhuma publicação foi excluída antes de ter sido lida e analisada. Todas passaram pelo processo de categorização na análise bibliométrica.

Primeiramente foi realizada a busca no banco de dados Scopus por ser esse uma das maiores fontes de literatura técnica e científica permitindo acesso a quantidade suficiente de informações para basear as análises e conclusões. Por ser o tema GovC intrinsicamente interdisciplinar, foi necessário escolher uma base também multidisciplinar.

Em sequência, foi realizada uma busca dirigida no banco de Teses e Dissertações. A Web of Science e a Google Acadêmico foram utilizadas para apoiar a busca de artigos completos, quando estes não estavam disponibilizados pela Scopus.

Diante disso, ao total foram identificadas 329 publicações que utilizam no seu título, palavras chave ou resumo, os descritores pré-selecionados como é demonstrado na Tabela 1. 
Tabela 1: Distribuição de publicações por descritor:

\begin{tabular}{|c|c|}
\hline \multicolumn{1}{|c|}{ Descritores } & Quantidade de Publicações \\
\hline "Governance of Knowledge" & 55 \\
\hline "Knowledge governance" & 273 \\
\hline "Governança do conhecimento" & 1 \\
\hline TOTAL & 329 \\
\hline
\end{tabular}

Elaborado pelos autores (2017).

Sobre estas 329 publicações foi realizada uma análise bibliométrica para a compreensão da evolução da produção científica sobre GovC para principalmente, mapear ONDE e com que FREQUÊNCIA o tema foi abordado pelas publicações. Ainda, poderá ser analisado o desenvolvimento dos estudos no campo da interdisciplinaridade e as tendências de pesquisas identificando as ondas de interesse e as áreas emergentes.

Após esta fase exploratória com a análise bibliométrica, foi realizada a análise descritiva sobre dezenove artigos aderentes ao tema, ou seja, que tratam de temas circundantes às discussões. Foram utilizados como critérios de exclusão: falta de conceituação para o termo governança do conhecimento e/ou; falta de inclusão do termo no título, palavras chave ou resumo e/ou; não acesso ao artigo completo e/ou; aplicação do termo "governança do conhecimento" como sinônimo de "gestão do conhecimento". Por exemplo, foi excluído da análise descritiva o segundo artigo mais citado, que foi o "External sources of knowledge, governance mode, and R\&D performance" de Fey e Birkinshaw (2005) publicado no Journal of Management. Mesmo ele tendo recebido 155 citações, além de não apresentar nem propor uma conceituação para o termo "governança do conhecimento", observou-se que tratava de governança específica de um setor corporativo, que seria a área de pesquisa e desenvolvimento (P\&D).

Como critério de inclusão, aplicou-se a determinação de incluir publicações referenciadas pelos artigos e livros estudados, desde que apresentassem conceituação, princípios e mecanismos de governança do conhecimento.

A quantidade e frequência de citações das publicações não foram motivo de exclusão ou de inclusão, apenas apoiou os pesquisadores na análise quanto a importância daquela publicação para a definição do termo. Assim, pode-se atender ao objetivo geral ao identificar a conceituação do construto Governança do Conhecimento proposta na literatura científica, como segue nas próximas seções.

\section{Análise bibliométrica: tamanho, crescimento e distribuição da bibliografia sobre governança do conhecimento}

Esta seção visa apresentar análise bibliométrica acerca dos 329 documentos encontrados em cada um dos descritores utilizados nas buscas: governance of kknowledge e knowledge governance. Uma primeira análise a se apresentar focaliza a distribuição das publicações ao longo dos anos. O que se pode perceber em relação a circulação do tema "Governança do conhecimento" é que apesar de haver variações nas publicações em relação ao descritor utilizado (Governance of Knowledge, ou, Knowledge Governance), houve crescente produtividade sobre o tema, a partir da virada do século (Figura 1 e Figura 2). 


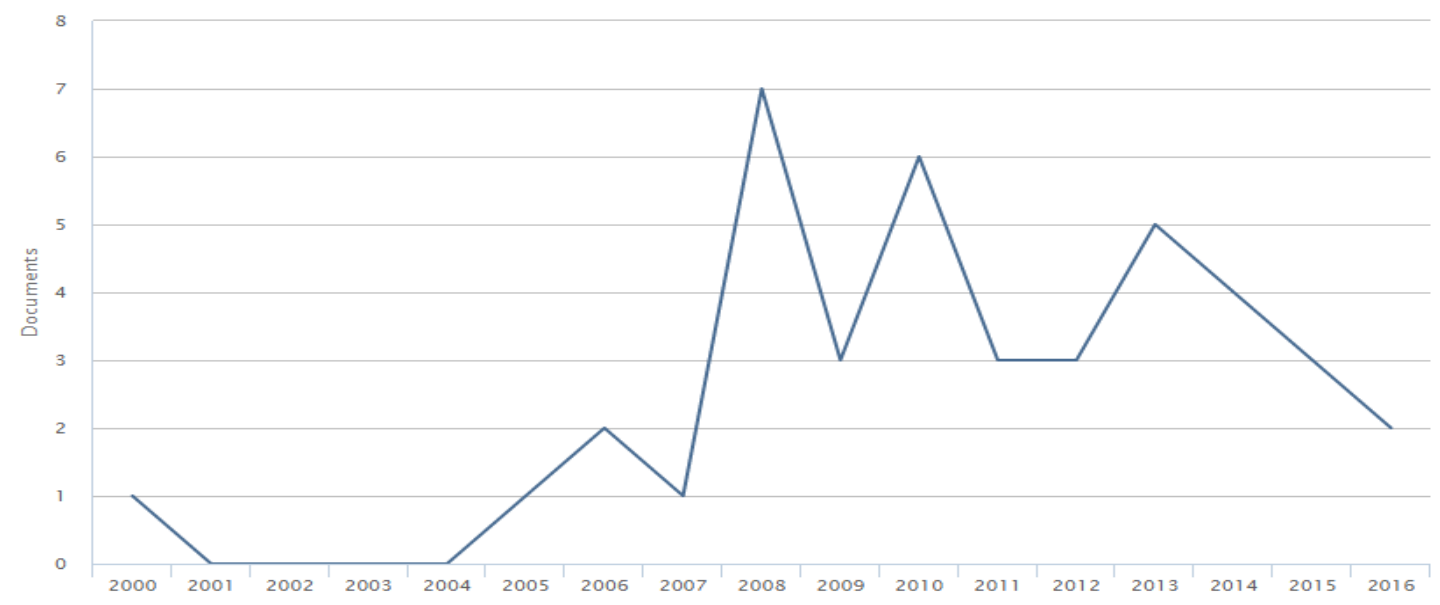

Figura 1: Linha do Tempo de publicações sobre Governance of Knowledge. Fonte: Scopus

A figura 2 retrata a distribuição das publicações com o descritor "knowledge governance",

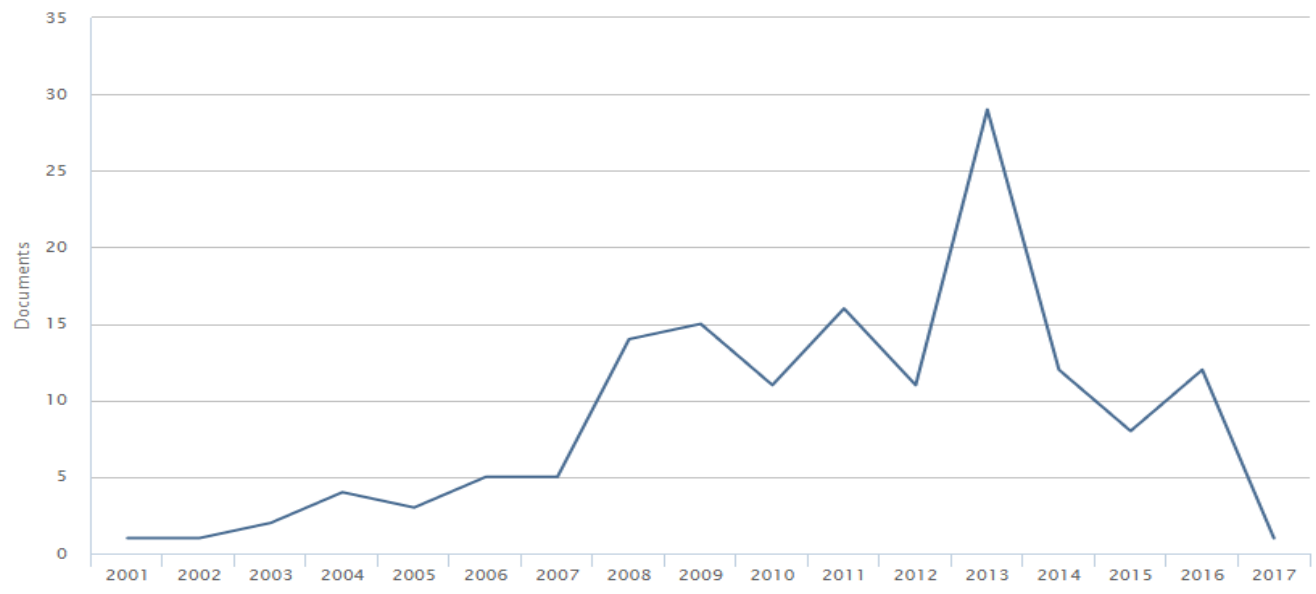

Figura 2: Linha do Tempo de publicações sobre Knowledge Governance Fonte: Scopus

Observa-se que, mesmo apresentando movimentos diferentes, a quantidade de publicações sobre os dois termos retrata o crescimento do interesse sobre o tema GovC a partir do ano de 2008, mantendo uma média relativamente alta até os dias atuais.

No que diz respeito aos autores que utilizam o descritor "governance of knowledge", destaca-se aqueles cuja produtividade alcançaram maiores índices (Quadro 1). 
Quadro 1: Autores que mais publicam no descritor Governance of Knowledge

\begin{tabular}{|c|c|c|}
\hline \multicolumn{3}{|r|}{ Governance of Knowledge } \\
\hline Autor & Quantidade & IES e País de origem \\
\hline Giebels, Diana & 3 & $\begin{array}{l}\text { Erasmus University Rotterdam, Department of Public Administration, } \\
\text { Rotterdam, Netherlands }\end{array}$ \\
\hline Van Buuren, Arwin & 3 & $\begin{array}{l}\text { Erasmus University Rotterdam, Department of Public Administration, } \\
\text { Rotterdam, Netherlands }\end{array}$ \\
\hline Bianchi, Marco & 2 & Fondazione Ugo Bordoni, Rome, Italy \\
\hline Brusoni, Stefano & 2 & Eidgenossische Technische Hochschule Zurich, Zurich, Switzerland \\
\hline Casalino, Nunzio & 2 & Libera Universita Internazionale degli Studi Sociali Guido Carli, Rome, Italy \\
\hline Draoli, Mauro & 2 & Agenzia per l'Italia Digitale, Rome, Italy \\
\hline Edelenbos, Jurian & 2 & $\begin{array}{l}\text { Erasmus University Rotterdam, Department of Public Administration and } \\
\text { Sociology, Rotterdam, Netherlands }\end{array}$ \\
\hline Gambosi, Giorgio & 2 & $\begin{array}{l}\text { Universita degli Studi di Roma Tor Vergata, Dipartimento di Ingegneria } \\
\text { dell'Impresa mario Lucertini, Rome, Italy }\end{array}$ \\
\hline Zyngier, Suzanne & 2 & La Trobe University, Melbourne, Australia \\
\hline
\end{tabular}

Elaborado pelos autores (2017).

Percebe-se que o constructo Governance of Knowledge, tem como destaque os pesquisadores europeus e um australiano. Todos os documentos encontrados por autor, encontram-se na seção de referências. Em relação as universidades, apontam-se a Erasmus University Rotterdam com quatro publicações e as demais instituições aparecem com duas frequências, e uma frequência, somente (Figura 3):

Erasmus University Rotterdam

DigitPA - Italian Agency for the Digitalization of Public Administration

Handelshoyskolen BI

Universita degli Studi di Torino

Universita degli Studi di Roma Tor Vergata

Universita degli Studi Guglielmo Marcon

Franklin College

Public Intellectual Property Resource for Agriculture

Center d'Études et de Recherches sur les Qualifications CEREQ

Telham Lodge

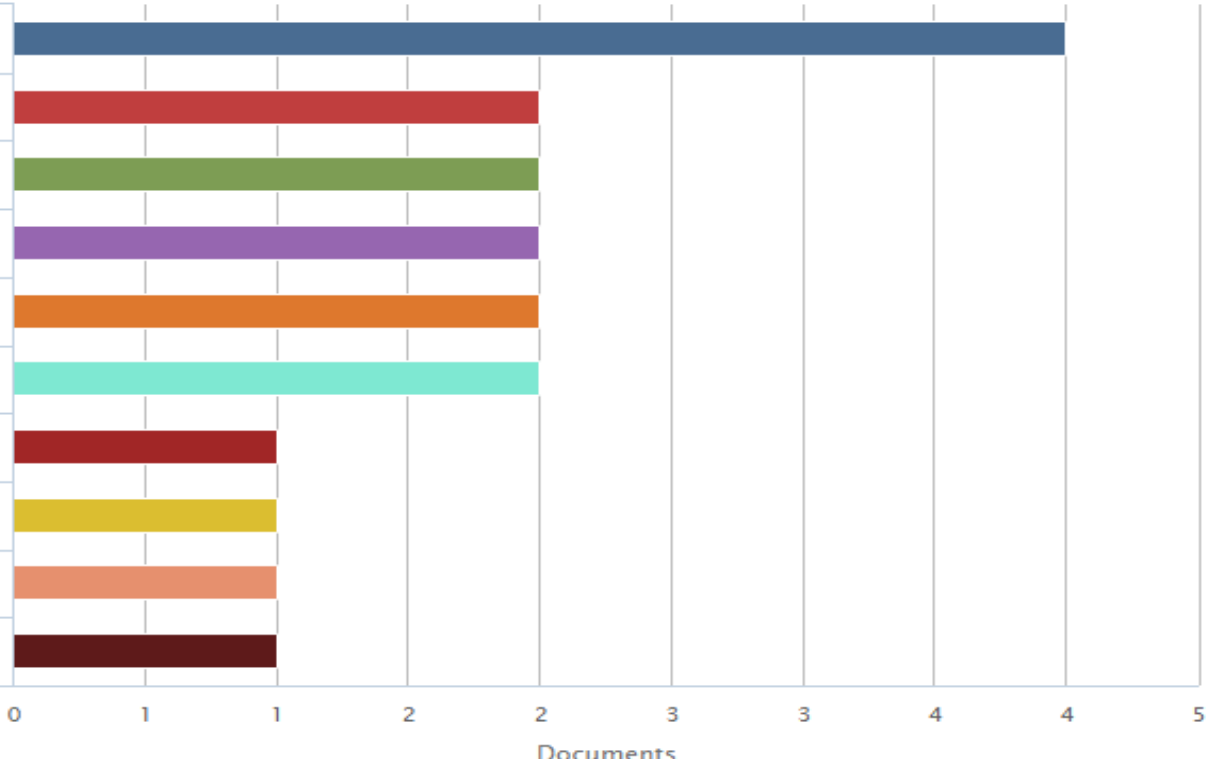


O termo Knowledge Governance apresentou quatro publicações brasileiras como a de Alves e Barbosa (2010) classificada na área da Ciência da Informação com uma citação na Scopus e o pesquisador Leonardo Burlamarqui da Universidade Estadual do Rio de Janeiro com um artigo (BURLAMAQUI, 2010), um livro (BURLAMAQUI, CASTRO E KATTEL, 2011) e um capítulo de livro (BURLAMAQUI, 2011) sobre o tema pela perspectiva da área da economia.

No Quadro 2, a seguir, são apresentados os autores que, no banco de dados Scopus, aparecem como os que mais publicam sobre o termo utilizando-se do termo Knowledge Governance.

Quadro 2: Autores que utilizam o termo Knowledge Governance.

\begin{tabular}{|c|c|c|}
\hline \multicolumn{3}{|r|}{ Knowledge Governance } \\
\hline Autor & Quantidade & Origem \\
\hline Antonelli, Cristiano & 14 & Universita degli Studi di Torino, Dipartimento di Economia, Torino, Italy \\
\hline Fassio & 5 & Lunds Universitet, Center for Innovation, Lund, Sweden \\
\hline Foss, Nicolai J. & 5 & $\begin{array}{l}\text { Norwegian School of Economics, Department of Strategy and Leadership, } \\
\text { Bergen, Norway }\end{array}$ \\
\hline Michailova, Snejina & 5 & University of Auckland, Auckland, New Zealand \\
\hline $\begin{array}{l}\text { Amidei, Federico } \\
\text { Barbiellini }\end{array}$ & 4 & Banca d'Italia, Economic Research Department, Rome, Italy \\
\hline Giebels, Diana & 4 & $\begin{array}{l}\text { Erasmus University Rotterdam, Department of Public Administration, Rotterdam, } \\
\text { Netherlands }\end{array}$ \\
\hline Grandori, Anna & 4 & Universita Bocconi, Milan, Italy \\
\hline Müller, Ralf O. & 4 & BI Norwegian Business School, Oslo, Norway \\
\hline Pedersen, Torben & 4 & Universita Bocconi, Department of Management and Technology, Milan, Italy \\
\hline Hovenga, Evelyn J S & 4 & eHealth Education Pty Ltd, , Australia \\
\hline Burlamaqui, Leonardo & 3 & Levy Institute, United States \\
\hline Chen, Le & 3 & Shanxi University, School of Life Science, Taiyuan, China \\
\hline Fong, P. S. W. & 3 & $\begin{array}{l}\text { Hong Kong Polytechnic University, Department of Building and Real Estate, } \\
\text { Hong Kong, China }\end{array}$ \\
\hline Garde, Sebastian. & 3 & Ocean Informatics, Dusseldorf, Germany \\
\hline Pemsel, Sofia & 3 & $\begin{array}{l}\text { Copenhagen Business School, Department of Organization, Copenhagen, } \\
\text { Denmark }\end{array}$ \\
\hline Vale, Mário & 3 & Universidade de Lisboa, Lisbon, Portugal \\
\hline
\end{tabular}

Elaborado pelos autores (2017).

Todos os documentos encontrados por autor, encontram-se na seção de referências. No que diz respeito às universidades, são os pesquisadores relacionados à Universita degli Studi di Torino que tem mais publicado sobre o tema (15 publicações), seguida da universidade de Copenhagen Business School com 13 publicações.

Assim, após realizar a análise bibliométrica do conjunto de publicações levantadas na base de dados Scopus, pode-se responder à questão que deu origem à revisão ao identificar que já existem 329 publicações que se utilizam do termo "governança do conhecimento" e suas variações em inglês, distribuídas entre diversos países, 
universidades e autores, não tendo uma concentração significativa em uma região ou grupo de pesquisa. Podese ainda, identificar o crescimento do interesse do tema para academia entre os anos de 2008 e 2013 , continuando em evidência até a atualidade.

\section{Análise descritiva: governança do conhecimento, conceito, princípios e mecanismos}

A leitura das publicações selecionadas, leva a compreensão do contexto de que, por demanda dos modelos de gestão propostos na década de 90 (MITROFF, 1994; GALBRAITH, 1997; NONAKA e TAKEUCHI, 1997; GOSHAL e BARTLET, 2000; HOLSAPPLE e SINGH, 2001), surge a proposta da Governança do Conhecimento (NOOTEBOOM, 2000) baseado no termo cunhado por Grandori (1997). Este modelo de governança avança sobre os limites do modelo de governança corporativa, ampliando os seus princípios de Transparência, equidade, prestação de contas e responsabilidade (OCDE, 2016; IBGC, 2014)

Tratar de Governança do Conhecimento é ser capaz de dialogar com três diferentes campos do saber: gestão do conhecimento, estudos organizacionais e estratégia e gestão de recursos humanos (Foss, 2007), pois está se falando de como e quais estratégias, estruturas e valores compartilhados são necessários para se gerenciar ativos do conhecimento.

Na publicação de Nooteboom (2000) identifica-se a gênese das discussões contemporâneas sobre governança do conhecimento e sua importância para apoio aos modelos de gestão que consideram o conhecimento como ativo, por isso é um artigo com 216 citações na Scopus. O autor entende a GovC como uma ferramenta de análise dos problemas e soluções relacionados com a troca e coprodução de conhecimento. O estudo baseia-se na correlação de três teorias: de Custos de Transação, de Troca Social e do Conhecimento. Nesse contexto, a governança consiste na combinação de mecanismos formais e relacionais, caracterizados pela transação ou relacionamento baseadas na lógica destas três teorias, que levam a perceber os custos e os esforços empreendidos pelas partes envolvidas para tentar minimizá-los.

Nesta lógica, a GovC, segundo Nooteboom (2000), se baseia em três princípios:
a) Intenção em conquistar um equilíbrio entre dependência e poder;
b) Criação de competências para manter ativa a capacidade absortiva de conhecimentos entre os colaboradores internos e externos;
c) Intenção de realizar "o melhor de sua capacidade".

Com estes desafios alcançados fica fácil, segundo Nooteboom (2000), motivar os participantes internos e externos para a cocriação e coprodução de novos conhecimentos essenciais ao sucesso pretendido, pelo fato de leva-los a "tomar os interesses dos outros no coração" (p.76-77 - tradução nossa).

Em 2001, Anna Grandori publica artigo intitulado "Neither hierarchy nor identity: Knowledge-governance mechanisms and the theory of the firm" no Journal of Management and Governance. Neste novo artigo, a autora resgata o termo que cunhou e aprofunda seus estudos sobre princípios e mecanismos de GovC intra e interorganizações apresentando diferentes casos relativos a transferência de conhecimento. Sobre os princípios, Grandori (2001) cita que, a governança deve ser contínua, além de buscar equilibrar e combinar os sistemas da organização. Quanto aos mecanismos, a autora acredita que os mecanismos de GovC podem ser avaliados a partir de dois critérios: o primeiro focado na possibilidade cognitiva de sustentar determinadas trocas de conhecimentos, e, o segundo, consiste nos custos atribuídos aos mecanismos, principalmente em casos onde mais do que um é aplicável. Ou seja, competências e estruturas para o compartilhamento e transferência de conhecimento, bem como os custos para que isso ocorra. 
Borghi (2006), mesmo não tendo muitas publicações sobre o termo ou não sendo um dos mais citados na base de dados Scopus, traz uma definição de governança que vale compartilhar. Para o autor, governança é um conjunto de transformações interrelacionadas com a intensificação e a difusão de práticas participativas para a otimização dos processos de troca entre diversos atores.

Neste mesmo ano de 2006, Peltokorpi e Tsuyuki (2006) descrevem em seu artigo, a importância da governança corporativa assumir a coordenação dos mecanismos que influenciam os processos de conhecimento, pois a sua não integração pode dificultar o compartilhamento do conhecimento levando a empresa a não alcançar os objetivos estratégicos pretendidos. Fugindo da instância da gestão do conhecimento, que parece ser a análise destes autores (PELTOKORPI e TSUYUKI,2006), aplica-se este conceito à GovC com base em Mayer (2006), o qual descreve que os mecanismos de Governança são responsáveis pelas decisões que afetam a criação e a proteção do conhecimento organizacional.

Uma das principais referências sobre GovC, é o pesquisador Nicolai Foss $(2007 ; 2011)$ e seus colaboradores, que analisam o termo pela visão da economia. Uma de suas publicações mais citadas (139) é a publicação "The emerging knowledge governance approach: Challenges and characteristics". Para Foss (2010) Governança do Conhecimento é resultante da interação da implantação dos mecanismos de governança corporativa e a gestão dos processos de conhecimento para a otimização dos resultados econômicos da organização. Em uma abordagem econômica, o autor vem examinando as inter-relações dos mecanismos de governança corporativa e as capacidades da organização em lidar com as transações baseadas em conhecimento como o compartilhamento, manutenção e criação desse fenômeno.

Grandori (2007) acrescenta ao conceito que este pode ser compreendido como um sistema organizacional colaborativo orientado à agregação de valores distintivos aos produtos, serviços e marcas da empresa, criando estruturas organizacionais que possibilitem a gestão de ativos intangíveis gerados nas inter-relações intra e inter organizacionais, constituídas por pessoas, processos e tecnologias.

O artigo "The governance of knowledge in project-based organizations" elaborado pelos autores Pemsel e Müller (2012) estuda mais profundamente as práticas de GovC, registrando a investigação de padrões destas práticas em organizações baseadas em projetos (PBOs). Os resultados confirmam que as práticas de GovC nas PBO's são impactadas por fatores estruturais e situacionais e que, os mecanismos de "governança informal" são tão os mais úteis do que os mecanismos formais quando se trata de processos de criação de conhecimento. Os autores acreditam que esses mecanismos informais parecem ser complexos para os executivos o que resulta na formação de barreiras às práticas produtivas de governança.

Durante a análise da publicação de Garde et. al. (2007) surgiu a dúvida se o autor estava se referindo a gestão do conhecimento ou a sua governança visto ele relaciona a Governança do Conhecimento à um conjunto de processos que possibilitam a criação, desenvolvimento, organização, compartilhamento, disseminação, utilização e manutenção contínua de arquétipos. Uma definição muito próxima da que se entende por gestão do conhecimento.

Também, gerou dúvidas o artigo "The business governance of localized knowledge: An information economics approach for the economics of knowledge" de Antonelli (2006), mas de qualquer maneira, os resultados desta pesquisa ajudam a identificar o papel da Governança para o controle e monitoramento da gestão do conhecimento. Antonelli acredita que algumas características do conhecimento exigem a existência de mecanismos de governança, como ele ser: dinâmico, heterogêneo e possuir níveis diversos de apropriação, tacitividade, imprevisibilidade e indivisibilidade. O autor identifica que nesta economia do conhecimento, é possível o desenvolvimento de ferramentas inteligentes para analisar os mecanismos de governança do conhecimento. A contribuição deste artigo ainda se destaca por sua conclusão quanto à variabilidade da performance da governança do conhecimento, definindo-a como híbrida, a qual é consolidada a partir de transações construídas coordenadamente e podem ser encontradas, segundo o autor, entre dois extremos, o da organização pura e o do mercado puro.

Amin e Cohendet (2011) definem GovC como uma abordagem voltada para governar a distribuição do conhecimento dentro e fora das organizações. A noção de governança está associada à noção de estratégia e 
estrutura de "comunidades de aprendizagem" para disseminação do conhecimento, favorecendo o ciclo da criação do conhecimento organizacional.

Na sequência, outros autores analisam governança do conhecimento pelas dimensões de pública e global. Para compreender o conceito de GovC por estas dimensões, vale rever a definição de governança proposta pelo Banco Mundial em seu documento Governance and Development (1992), onde define Governança como: "exercício de autoridade, controle, gerenciamento e poder de governo". Este documento afirma ainda que: "é a forma como o poder é exercido na administração dos recursos econômicos e sociais de um país para o desenvolvimento" (WORLD BANK, 1992, p. 3).

Aplicando-se esta definição de governança à GovC surge as questões relacionadas à propriedade intelectual. Por exemplo, a publicação "The global governance of knowledge: Patent offices and their clientes", escrito por Drahos, em 2010, teve o objetivo de analisar a atuação dos escritórios de marcas e patentes de diversos países no desenvolvimento de "políticas de agência" voltadas para os clientes. Segundo o autor, esses escritórios fazem parte da governança privada globalmente integrada, que serve aos interesses das organizações multinacionais. Ao seguir nesta argumentação, o autor reconhece a GovC como uma abordagem inovadora à administração de patentes, pois permitiria, aos países desenvolvidos e em desenvolvimento, retomarem o espírito público do contrato social de patentes.

Burlamarqui $(2010,2011)$ avança sobre a dimensão pública de GovC, trazendo suas premissas relacionadas às "políticas de inovação, de concorrência e de propriedade intelectual de uma perspectiva evolucionária" (2011, p.1). Como destaca o autor, o termo Governança do Conhecimento pode ter uma abordagem analítica e orientada para uma política alternativa de interesse público referentes aos problemas relacionados com a produção, apropriação e difusão do conhecimento. Seguindo por esta abordagem da governança pública, com seus colaboradores Castro e Kattel, Burlamaqui (2011) percebe a governança do conhecimento como uma solução a ser aplicada para a diminuição dos conflitos entre os interesses públicos e privados quanto ao regime de direitos de propriedade intelectual internacional. Os autores argumentam que a Organização Mundial do Comércio (OMC), ao liderar o regime de direitos de propriedade intelectual internacional, tem privilegiado os interesses privados em detrimento dos interesses públicos no acesso a difusão do conhecimento e da inovação, mesmo quando se trata da área de saúde. Faz-se necessário então, a implantação de uma governança, capaz de gerar cooperação e orientar ações coordenadas de política internacional para o bem coletivo, além do empresarial. Ou seja, os autores sugerem a Governança do Conhecimento como uma nova abordagem de gestão da propriedade intelectual oriundas de pesquisa e desenvolvimento. Um modelo que reconheceria a importância do lucro para as organizações privadas, mas reafirmaria o interesse público.

Em resumo, pode-se levar em consideração para melhor compreender o constructo GovC, em inglês Governance Knowledge ou Knowledge of Governance, as seguintes publicações (Quadro 3):

Quadro 3: Definição de Governança do Conhecimento.

\begin{tabular}{|c|l|l|}
\hline Autor (data) & \multicolumn{1}{|c|}{ Definição } & Dimensão de análise \\
\hline \multirow{2}{*}{ Nooteboom (2000) } & $\begin{array}{l}\text { Uma combinação de mecanismos formais e relacionais, caracterizados pela } \\
\text { transação ou relacionamento baseadas na percepção dos custos e dos } \\
\text { esforços empreendidos pelas partes envolvidas para tentar minimizá-los } \\
\text { com o objetivo maior de motivar os participantes internos e externos para a } \\
\text { cocriação e coprodução de novos conhecimentos essenciais ao sucesso da } \\
\text { organização. }\end{array}$ & Organizacional \\
\hline Grandori (2001) & $\begin{array}{l}\text { Governança contínua, com ocorrência intra e inter organizações, utilizando- } \\
\text { se de mecanismos para equilibrar e combinar os sistemas da organização. }\end{array}$ & Organizacional \\
\hline
\end{tabular}


Quadro 3: Definição de Governança do Conhecimento (continuação).

\begin{tabular}{|c|c|c|}
\hline Autor (data) & Definição & Dimensão de análise \\
\hline Antonelli (2006) & $\begin{array}{l}\text { Uma governança híbrida para o controle e monitoramento da gestão do } \\
\text { conhecimento, a qual é consolidada a partir de transações construídas } \\
\text { coordenadamente }\end{array}$ & Organizacional \\
\hline $\begin{array}{l}\text { Peltokorpi, C. e } \\
\text { Tsuyuki (2006) }\end{array}$ & $\begin{array}{l}\text { Coordenação dos mecanismos que influenciam os processos de } \\
\text { conhecimento - hierarquia pautada em consenso, busca de práticas de } \\
\text { recursos humanos e medidas de controle, desempenho e saída do } \\
\text { conhecimento. }\end{array}$ & Organizacional \\
\hline Mayer (2006) & $\begin{array}{l}\text { Instância responsável pelas decisões que afetam a criação e a proteção do } \\
\text { conhecimento organizacional. }\end{array}$ & Organizacional \\
\hline $\begin{array}{l}\text { Garde et. al. } \\
(2007)\end{array}$ & $\begin{array}{l}\text { Compreende um conjunto de processos que possibilitam a criação, } \\
\text { desenvolvimento, organização, compartilhamento, disseminação, utilização } \\
\text { e manutenção contínua de arquétipos. }\end{array}$ & Organizacional \\
\hline $\begin{array}{l}\text { Foss } \\
(2007 ; 2010)\end{array}$ & $\begin{array}{l}\text { Resultado da interação da implantação dos mecanismos de governança } \\
\text { corporativa e a gestão dos processos de conhecimento para a otimização } \\
\text { dos resultados econômicos da organização. }\end{array}$ & Organizacional \\
\hline $\begin{array}{l}\text { Amin e Cohendet } \\
(2010)\end{array}$ & $\begin{array}{l}\text { Abordagem voltada para a distribuição do conhecimento dentro e fora das } \\
\text { organizações. A noção de governança está associada à noção de } \\
\text { "comunidades de aprendizagem" para disseminação do conhecimento. }\end{array}$ & Organizacional \\
\hline $\begin{array}{l}\text { Alves e Barbosa } \\
\text { (2010) }\end{array}$ & $\begin{array}{l}\text { "Envolve o uso e o controle adequado da estrutura organizacional, de } \\
\text { organogramas de trabalho, dos sistemas de recompensa, dos sistemas de } \\
\text { informação, dos procedimentos operacionais padrão e de outros } \\
\text { mecanismos de coordenação", e ainda, deve ser reelaborada para passar a } \\
\text { "incorporar em seu escopo fenômenos motivacionais e cognitivos } \\
\text { associados à produção e ao compartilhamento do conhecimento" (2010, } \\
\text { p.11). }\end{array}$ & Organizacional \\
\hline Drahos (2010) & $\begin{array}{l}\text { Abordagem inovadora à administração de patentes para que os países } \\
\text { desenvolvidos e em desenvolvimento, retomem o espírito público do } \\
\text { contrato social de patentes. }\end{array}$ & Global \\
\hline $\begin{array}{l}\text { Burlamarqui } \\
(2010,2011)\end{array}$ & $\begin{array}{l}\text { Abordagem analítica e orientada para uma política alternativa de interesse } \\
\text { público referentes aos problemas relacionados com a produção, apropriação } \\
\text { e difusão do conhecimento. }\end{array}$ & Público \\
\hline $\begin{array}{l}\text { Burlamaqui, } \\
\text { Castro e Kattel, } \\
\text { (2011) }\end{array}$ & $\begin{array}{l}\text { Abordagem de gestão da propriedade intelectual oriundas de pesquisa e } \\
\text { desenvolvimento, que reconhece a importância do lucro para as } \\
\text { organizações privadas, mas reafirmaria o interesse público. } \\
\text { Solução a ser aplicada para a diminuição dos conflitos entre os interesses } \\
\text { públicos e privados quanto ao regime de direitos de propriedade intelectual } \\
\text { internacional, por ser capaz de gerar cooperação e orientar ações } \\
\text { coordenadas de política internacional para o bem coletivo, além do } \\
\text { empresarial. }\end{array}$ & Público e Global \\
\hline
\end{tabular}

Elaborado pelos autores (2017). 
Pela dimensão de análise organizacional, reconhecendo a contribuição da conceituação dada por Nooteboom (2000) e Foss (2010), para este artigo define-se a Governança do Conhecimento como um conjunto de mecanismos formais e relacionais gerados pela governança corporativa e da gestão dos processos de conhecimento para a otimização dos resultados econômicos da organização.

Seguindo na análise sobre as publicações selecionadas, percebeu-se que as publicações mais recentes sobre o tema não parecem ter mais a preocupação de defini-lo, mas de aprofundar os mecanismos para a sua implantação.

Pelos estudos analisados, identifica-se que o modelo Governança do Conhecimento, revisita os outros modelos de governança (corporativa, pública e global) à luz da teoria da aprendizagem, da firma e da gestão do conhecimento. Este novo modelo se baseia na intenção da empresa em conquistar um equilíbrio entre dependência e poder; no desenvolvimento de competências para manter ativa a capacidade absortiva de conhecimentos entre os colaboradores internos e externos e; inclusive, na intenção de realizar o melhor de sua capacidade técnica. E para tal conquista, as publicações vem tentando identificar quais os princípios que devem ser respeitados pelas organizações para que a Governança do Conhecimento seja efetiva.

Para o OCDE, os "Princípios são desenvolvidos com base no entendimento de que as políticas de governo das sociedades têm um papel importante a desempenhar na prossecução de objetivos económicos mais amplos relativamente à confiança dos investidores e formação e alocação de capital”. Respeita-se os princípios da governança corporativa e aplica-se a compreensão aos princípios da governança do conhecimento, busca-se os mecanismos para que estes princípios sejam respeitados na prática de gestão do conhecimento.

Em qualquer das dimensões de análise - global, pública, corporativa e de conhecimento, identifica-se que para se implantar a governança faz-se necessário a integração de diferentes competências organizacionais para a elaboração de políticas e estruturas corporativas voltadas a governar com foco no respeito à coletividade e a sustentabilidade econômica, social e ambiental. Para tal, faz-se necessária alguns mecanismos como os sistemas de autoridade, de liderança e de incentivos formais; de comunicação interna e com stakeholders; de características da cultura para a transparência e controles, contratos psicológicos para a confiança e compartilhamento; e a construção social de sentido (FOSS e KLEIN, 2008).

Ainda, Anna Grandori (2008) na origem das discussões sobre Governança do Conhecimento, já mencionava a importância de mecanismos de redes intra e Inter organizacionais, destacando que as organizações tornamse altamente eficazes e intensivas em conhecimentos inovadores, ao se descentralizarem, passando a ser governadas por comunidades e equipes de projetos que potencializam o compartilhamento dos direitos de propriedade, ao invés de ilhas de conhecimentos tácitos centralizados. Ao se trabalhar com redes, os mecanismos de tomada de decisão passam a ocorrer em contínua e crescente colaboração, em um modelo mais de governança multi nível. Para tal, faz-se necessário a profissionalização da gestão, saindo de uma gestão intuitiva de poucos decisores, para uma gestão compartilhada com a colaboração de todos. Um novo modelo, também exigirá mecanismos de gestão compartilhada, como a transparência quanto às descrições de tarefas e cargos, a qualidade e a abrangência dos sistemas de informação e conhecimentos; trabalho em equipe.

Em um primeiro momento, Grandori (2009) sinaliza a poliarquia (gestão participativa e inclusiva) ao invés da hierarquia, e evidenciando a rede como basilar nesses processos. Esta sugestão vem ao encontro à necessidade de uma combinação de diferentes mecanismos de governança relacionados à estrutura e aos incentivos para o compartilhamento do conhecimento e a participação em redes de aprendizagem. Para a Governança destas redes propiciar a aprendizagem, a autora (Grandori, 2008) destaca a importância de mecanismos que potencialize a capacidade absortiva organizacional; formação de parcerias; a construção de laços de confiança para propiciar a aproximação e a compreensão do outro aumentando o nível de empatia; atenção a linguagem comum para a comunicação ser efetiva em diminuir distâncias cognitivas e potencializar novos relacionamentos. (NOOTEBOOM, 1996; GRANORI, 2008).

Complementando a listagem sobre os mecanismos que deverão ser utilizados para a governança do conhecimento, esta pesquisa identificou duas publicações do ano de 2006 de Foss, e Peltokorpi e Tsuyuki e uma de 2010 de Alves e Barbosa. Ao analisar os estudos de Foss (2006), encontra-se a questão central da sua 
abordagem para Governança de Conhecimento, que seja o como os mecanismos de governança influenciam os processos de coprodução, difusão e apropriação do conhecimento. Para o autor, pela visão econômica, os mecanismos da governança do conhecimento são os custos e os riscos de transação de transferência do conhecimento, como a frequência do uso, a incerteza e a especificidade dos ativos de conhecimento, somando-se as despesas com a inspeção, o acompanhamento do desempenho de entrada, e outros custos com a transformação e aplicação do ativo.

Somam-se ainda, os mecanismos determinados por Peltokorpi e Tsuyuki (2006) para a governança do conhecimento, que são: hierarquia pautada em consenso, busca de práticas de recursos humanos e medidas de controle, do desempenho e da saída do conhecimento.

Pela análise da governança do conhecimento sob a luz da dimensão organizacional, ainda surgem Alves e Barbosa (2010) que determinam que a "governança do conhecimento, envolve o uso e o controle adequado da estrutura organizacional, de organogramas de trabalho, dos sistemas de recompensa, dos sistemas de informação, dos procedimentos operacionais padrão e de outros mecanismos de coordenação", e ainda, deve ser reelaborada para passar a "incorporar em seu escopo fenômenos motivacionais e cognitivos associados à produção e ao compartilhamento do conhecimento” (2010, p.11).

Quadro 4: Mecanismos de Governança do Conhecimento.

\begin{tabular}{|c|c|}
\hline Categorias de Governança Corporativa & Mecanismos de Governança do Conhecimento \\
\hline Transparência & $\begin{array}{l}\text { 1. Formação de parcerias internas e externas com base em uma cultura } \\
\text { de transparência. } \\
\text { 2. Formação de redes intra e Inter organizacionais com comunicação } \\
\text { efetiva para diminuir distâncias cognitivas e potencializar novos } \\
\text { relacionamentos. } \\
\text { 3. Práticas de gestão de pessoas para a construção de laços psicológicos } \\
\text { de confiança e compartilhamento que propiciem a aproximação e a } \\
\text { compreensão do outro pelo aumento do nível de empatia. } \\
\text { 4. Incentivos formais à GC. }\end{array}$ \\
\hline Equidade & $\begin{array}{ll}\text { 5. } & \text { Compartilhamento dos direitos de propriedade. } \\
\text { 6. } & \text { Promoção da capacidade absortiva organizacional. } \\
\end{array}$ \\
\hline Prestação de Contas & $\begin{array}{l}\text { 7. Medidas de desempenho e monitoramento para controle de custos e } \\
\text { dos riscos de transação de saída e transferência do conhecimento }\end{array}$ \\
\hline Responsabilidade & $\begin{array}{l}\text { 8. Gestão descentralizada, coordenada por comunidades e equipes de } \\
\text { projetos; } \\
\text { 9. Promoção da inclusão para a participação e a colaboração. } \\
\text { 10. Sistemas de autoridade e de liderança, com a hierarquia pautada em } \\
\text { consenso com a construção social de sentido para a tomadas de } \\
\text { decisão. }\end{array}$ \\
\hline
\end{tabular}

Elaborado pelos autores (2017)

Em resumo (Quadro 4), com base na literatura estudada, pode-se definir que, para a organização respeitar os princípios da governança corporativa, capacitando-se a implantar, avaliar, monitorar e controlar os custos e resultados dos processos de gestão dos conhecimentos essenciais para o sucesso da estratégia organizacional por meio da sustentabilidade de sua cadeia de valor, deve-se implantar mecanismos de Governança do Conhecimento, principalmente quanto aos processos relacionados ao compartilhamento, troca e transferência do conhecimento organizacional.

Desta forma, pode-se representar em uma síntese (Figura 4) dos três níveis de mecanismos formais e relacionais que deverão ser governados. 


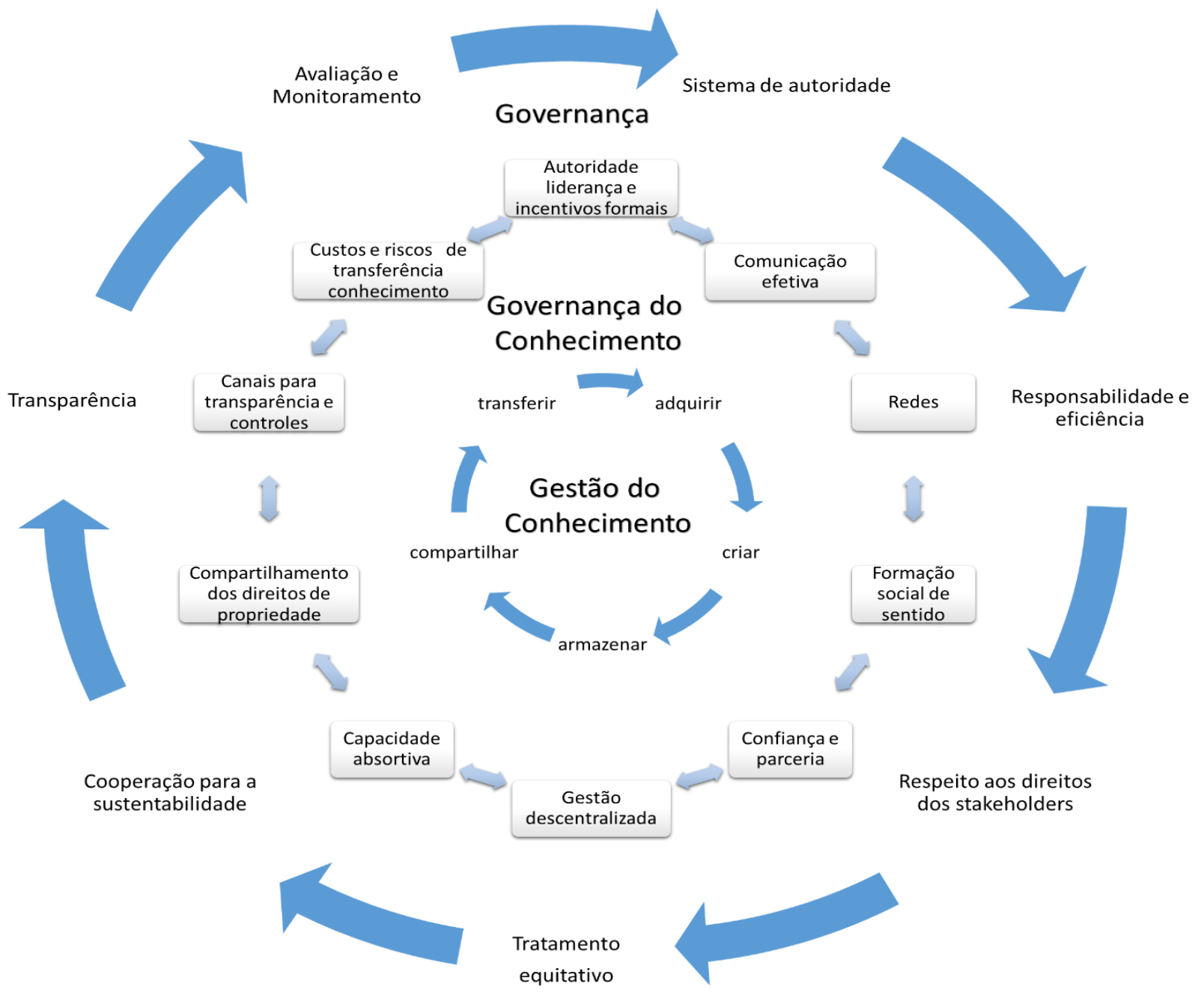

Figura 4: Síntese da Governança do Conhecimento

Fonte: Elaborados pelos autores (2017).

Como compreendem Foss e Michailova (2009) em seu livro "Knowledge Governance: Processes and Perspectives", estes mecanismos de GovC concernem aos campos da administração geral, gestão de recursos humanos, gestão de capital intelectual, teoria da inovação, gestão estratégica, tecnologia e negócios internacionais, dentre outros. Assim, para governa-los será necessária uma visão interdisciplinar consistente que transpasse os processos de gestão do conhecimento, alcançando instâncias organizacionais mais abrangentes.

A lista dos dez mecanismos de GovC identificados nesta revisão da literatura, deverá ser implantada para criar capacidades organizacionais que propiciem a implantação da GC, ou melhor, promovam a motivação para a GC, além de seu monitoramento e a avaliação de seus processos. Em resumo, a GovC vai além da GC ao ser vista como a implantação de mecanismos de governança corporativa para a implantação, avaliação e controle dos processos de conhecimento, principalmente no que tange os processos de compartilhamento, troca e transferência do conhecimento.

\section{Conclusões}

Após realizar uma sistemática revisão da literatura, e as respectivas análises bibliométrica e descritiva das publicações selecionadas, percebeu-se que o tema GovC é um constructo emergente no campo da gestão do conhecimento. E há diferentes olhares sobre o termo, por ser este um constructo complexo que pode ser explorado por visões de mundo distintas, e que, por ser interdisciplinar atravessa os campos de gestão do 
conhecimento, estudos organizacionais, estratégia e gestão de recursos humanos (FOSS, 2003; FOSS, 2007; FOSS e KLEIN, 2008).

Como conclusão desta pesquisa, pode-se considerar que a Governança do Conhecimento (GovC), mais do que o seu conceito, deve-se considerar os seus principais objetivos, que são: (i) privilegiar o conhecimento organizacional de modo estratégico; (ii) garantir a sobrevivência e a perenidade da organização por meio da gestão dos ativos do conhecimento; (iii) controlar e monitorar os processos de compartilhamento, troca e transferência de conhecimentos e tecnologias e; (iv) delinear estratégias, políticas e práticas de GC.

Com a implementação da GovC, a organização conseguirá consolidar paradigmas de gestão colaborativa com o objetivo de compartilhar, trocar e transferir conhecimentos desde o nível intra organizacional até o inter organizacional e, em redes de aprendizagem.

Nesta linha de argumentação, aponta Foss (2003, 2007), que para se gerenciar os processos de conhecimento é indispensável escolher uma estrutura de governança corporativa que propicia a colaboração, compartilhamento em multi níveis. Inclusive, há a necessidade de definir "mecanismos de governança e de coordenação [...], de modo a influenciar favoravelmente os processos de transferência, compartilhamento e criação do conhecimento" (FOSS, 2003, p.11).

Por fim, chega-se à conclusão que, GovC deve ser implementada nas organizações brasileiras para que promovam o equilíbrio entre dependência e poder por meio de um conjunto de mecanismos formais e relacionais para a otimização dos resultados econômicos da organização.

E, como ainda não há um modelo de governança do conhecimento a ser seguido, este estudo oferece subsídios para a elaboração de um modelo que reconheça o conhecimento como valor crítico para o sucesso da organização, lhe oferecendo apoio quanto as princípios e mecanismos a serem respeitados.

\section{Referências}

ACHA, V. BRUSONI, S. The changing governance of knowledge in avionics. Economics of Innovation and New Technology, 2008.

ADAMS, P. BRUSONI, S. MALERBA, F. The long-term evolution of the knowledge boundaries of firms: Supply and demand perspectives. The Third Industrial Revolution in Global Business, 2010.

ALVES, A. BARBOSA, R.R. Influences and barriers to information sharing: A theoretical perspective. Ciencia da Informação, 2010.

AMIN, A., COHENDET, P. Architectures of Knowledge: Firms, Capabilities, and Communities. Architectures of Knowledge: Firms, Capabilities, and Communities pp. 1-194, 2011.

ANTONELLI, C. A. Schumpeterian growth model: wealth and directed technological change. Journal of Technology Transfer, 2016.

ANTONELLI, C. The new economics of the university: A knowledge governance approach. Journal of Technology Transfer, 2008.

ANTONELLI, C. Technological knowledge as an essential facility. Journal of Evolutionary Economics, 2007.

ANTONELLI, C. The business governance of localized knowledge: An information economics approach for the economics of knowledge. Industry and Innovation, 2006.

ANTONELLI, C. FASSIO, C. Globalization and the Knowledge-Driven Economy. Economic Development Quarterly, 2016.

ANTONELLI, C. Knowledge Governance: Pecuniary Knowledge Externalities and Total Factor Productivity Growth. Economic Development Quarterly, 2013.

ANTONELLI, C. AMIDEI, F.B. FASSIO, C. Corrigendum to "The mechanisms of knowledge governance: State owned enterprises and Italian economic growth, 1950-1994". Structural Change and Economic Dynamics, 2015. 
ANTONELLI, C. AMIDEI, F.B. FASSIO, C. The mechanisms of knowledge governance: State owned enterprises and Italian economic growth, 1950-1994. Structural Change and Economic Dynamics, 2014.

ANTONELLI, C. AMIDEI, F.B. The dynamics of knowledge externalities: Localized technological change in Italy, 2011.

ANTONELLI, C. CALDERINI, M. The governance of knowledge compositeness and technological performance: The case of the automotive industry in Europe. Economics of Innovation and New Technology, 2008.

ANTONELLI, C. FASSIO, C. The heterogeneity of knowledge and the academic mode of knowledge governance: Italian evidence in the first part of the 20th century. Science and Public Policy, 2014.

ANTONELLI, C. PATRUCCO, P.P. QUATRARO, F. The governance of localized knowledge externalities. International Review of Applied Economics, 2008.

ANTONELLI, C. TEUBAL, M. Venture capitalism as a mechanism for knowledge governance. The Capitalization of Knowledge: A Triple Helix of University-Industry-Government, 2010.

ASSUMPÇÃO, T. Visão sistêmica relaciona conhecimento e ativos intangíveis. FNQ, 2008. Disponível em: <www.fnq.org.br/site/ltemID=1032/369/default.aspx>. Acesso em: 23 out. 2011.

BIANCHI, M. CASALINO, N. DRAOLI, M. GAMBOSI, G. An innovative approach to the governance of e-government knowledge management systems. Information Systems: Crossroads for Organization, Management, Accounting and Engineering: ItAIS: The Italian Association for Information Systems, 2013.

BIANCHI, M. CASALINO, N. DRAOLI, M. GAMBOSI, G. An innovative approach to the governance of E-government knowledge management systems. Information Systems: Crossroads for Organization, Management, Accounting and Engineering: ItAIS: The Italian Association for Information Systems, 2012.

BORGHI, V. "Tra cittadini e istituzioni. Riflessioni sull'introduzione di dispositivi partecipativi nelle pratiche istituzionali locali", in Rivista delle politiche sociali, $n^{\circ} 2$, Ediesse, Roma, 2006.

BURLAMAQUI, L. Knowledge Governance Innovation and Development. Revista de Economia Política (Impresso), v. 30, p. 560-580, 2010.

BURLAMAQUI, L. Knowledge Governance: An Analytical Approach and its Policy Implications. In: Leonardo Burlamaqui, Ana Celia Castro, Rainer Kattel. (Org.). Knowledge Governance - Reasserting the Public Interest. 1ed.Londres: Anthem Press, 2011 , v. 1, p. 3-27.

BURLAMAQUI, L. CASTRO, A.C.; KATTEL, R. Knowledge Governance - Reasserting the Public Interest. Anthem Press, 2011.

BYRD, T.A. MARKLAND, R.E. KARWAN, K.R. PHILIPOOM, P.R. An object-oriented rule-based design structure for a maintenance management system, 1966.

CABRITA, R. O capital intelectual: a nova riqueza das organizações. Revista Digital do Instituto de Formação Bancária. European Distance Education Network. Jun. 2004. Disponível em: <www.ifb.pt/publicacoes/info_57/artigo03_57.htm>. Acesso em: 12 jun. 2004.

CADBURY, Adrian. The Financial Aspects of Corporate Governance. The Committee on the Financial Aspects of Corporate Governance and Gee and Co. Ltd. London: Committee on the Financial Aspects of Corporate Governance. 1992.

CARVER, J. On Board Leadership. Jossey-Bass Wiley, J-B Carver Board Governance Series v.1. 2001.

CHEN, L. FONG, P.S.W. Visualizing evolution of knowledge management capability in construction firms. Journal of Construction Engineering and Management, 2013.

CHEN, L. FONG, P.S.W. Revealing performance heterogeneity through knowledge management maturity evaluation: A capability-based approach. Expert Systems with Applications, 2012.

CURTY, R.G. O Fluxo da informação tecnológica no projeto de produtos em indústrias de alimentos. 2005. 249 f. Dissertação (Mestrado em Ciência da Informação) - Programa de Pós-Graduação em Ciência da Informação, Universidade Federal de Santa Catarina. Florianópolis.

DRAHOS, P. The global governance of knowledge: Patent offices and their clients. The Global Governance of Knowledge: Patent Offices and their Clients pp. 1-351, 2010.

FREIRE, P.S. Aumente a qualidade e quantidade de suas publicações científicas: manual para projetos e artigos científicos. Curitiba: Editora CRV. 2013.

FREIRE, P. S., SOARES, A.P., NAKAYAMA,K.M, SPANHOL, F. J. Processo de Profissionalização com a implantação de Boas Práticas de Governança Corporativa para a Abertura de Capital (IPO) em Empresa Brasileira com Gestão de Tipo Familiar In: XXVIII Encontro Nacional de Engenharia de Produção, Rio de Janeiro, 2008, 
FREIRE, P. S., NAKAYAMA, K.M., SPANHOL, F. J. Compartilhamento do Conhecimento: Grupo Colaborativo um Caminho para o Processo de Aprendizagem Organizacional In: Gestão de Pessoas.1 ed. Florianópolis: Pandion, 2010.

FREIRE, P.S. Aumente a qualidade e quantidade de suas publicações científicas: manual para projetos e artigos científicos. Curitiba: Editora CRV. 2014.

FONG, P.S.W. CHEN, L. Governance of learning mechanisms: Evidence from construction firms. Journal of Construction Engineering and Management, 2012.

FOSS, N.J. The Knowledge Governance Approach, 2005.

FOSS, N. J. 2007 'The emerging knowledge governance approach: Challenges and characteristics,' Organization 14: $29-52$.

FOSS, K. FOSS, N.J. Managerial Authority When Knowledge is Distributed: A Knowledge Governance Perspective. Knowledge Governance: Processes and Perspectives, 2009.

FOSS, N.J. KLEIN, P.G. The theory of the firm and its critics: A stocktaking and assessment. New Institutional Economics: A Guidebook, 2008

FOSS, N. J .;K. HUSTED, S. MICHAILOVA, AND T. PEDERSEN. 2003. GoverningKnowledge Processes: Theoretical Foundations and Research Opportunities. Workingpaper no. 1, Center for Knowledge Governance, Copenhagen Business School.

FOSS, N.J. MICHAILOVA, S. Knowledge Governance: What have We Learned? and Where are We Heading? Knowledge Governance: Processes and Perspectives, 2009.

GALBRAITH, J. Projetando a organização inovadora. In: STARKEY,K. Como as organizações aprendem: relatos de sucesso da grandes organizações. SP: Futura,1997, p.190-218

GARDE, S. Clinical knowledge governance: the international perspective. Studies in health technology and informatics, 2013.

GARDE, S. KNAUP, P. HOVENGA, E.J.S. HEARD, S. Towards semantic interoperability for electronic health records: Domain knowledge governance for openEHR archetypes. Methods of Information in Medicine, 2007.

GIEBELS, D. TEISMAN, G.R. Towards ecosystem-based management for Mainports: A historical analysis of the role of knowledge in the development of the Rotterdam harbor from 1827 to 2008. Ocean and Coastal Management, 2015.

GIEBELS, D. VAN BUUREN, A. EDELENBOS, J. Knowledge governance for ecosystem-based management: Understanding its context-dependency. Environmental Science and Policy, 2016.

GIEBELS, D. VAN BUUREN, A. Edelenbos, J. Using knowledge in a complex decision-making process - Evidence and principles from the Danish Houting project's ecosystem-based management approach. Environmental Science and Policy, 2015

GIEBELS, D. VAN BUUREN, A. EDELENBOS, J. Ecosystem-based management in the Wadden Sea: Principles for the governance of knowledge. Journal of Sea Research, 2013.

GIEBELS, D. DE JONGE, V.N. Making ecosystem-based management effective: Identifying and evaluating empirical approaches to the governance of knowledge. Emergence: Complexity and Organization, 2014.

GOLDMAN, F. Governança do conhecimento e gestão do conhecimento organizacional. Revista Gestão \& Tecnologia, Pedro Leopoldo, v. 1, n. 1, p. 4-16, fev. 010 (b).

GOODERHAM, P. MINBAEVA, D.B. PEDERSEN, T. Governance Mechanisms for the Promotion of Social Capital for Knowledge Transfer in Multinational Corporations. Journal of Management Studies, 2011.

GOSHAL, S; BARTLETT,C. A organização individualizada. RJ: Campus,2000.

GRANDORI, A. Epistemic economics and organization: Forms of rationality and governance for a wiser economy. Epistemic Economics and Organization: Forms of Rationality and Governance for a Wiser Economy, 2013.

GRANDORI, A. FURLOTTI, M. Facio ut facias: Associational contracts for innovation. International Studies of Management and Organization, 2009.

GRANDORI, A. Poliarchic Governance and the Growth of Knowledge. Knowledge Governance: Processes and Perspectives, 2009.

GRANDORI, ANNA (2001) 'Neither Hierarchy nor Identity: Knowledge Governance Mechanisms and the Theory of the Firm,' Journal of Management and Governance 5:381-399.

GRANDORI, ANNA (1997) 'Governance Structures, Coordination Mechanisms and Cognitive Models,' Journal of Management and Governance 1: 29-42. 
HELOU, A. R. H. A. Método de gestão integrada de riscos no contexto da administração pública. Dissertação (Dissertação de Mestrado). 208 f. Programa de Pós-Graduação em Engenharia e Gestão do Conhecimento da Universidade Federal de Santa Catarina. Santa Catarina: UFSC, 2009.

HOLSAPPLE,S.W; SINGH,M. The knowledge chain model: activities for competitiveness. Expert Systems with application , v.20, p.77-98, 2001

HOVENGA, E. GARDE, S. HEARD, S. Nursing constraint models for electronic health records: A vision for domain knowledge governance. International Journal of Medical Informatics, 2005.

HUSTED, K. MICHAILOVA, S. MINBAEVA, D.B. PEDERSEN, T. Knowledge-sharing hostility and governance mechanisms: An empirical test. Journal of Knowledge Management, 2012.

IBGC. Introdução às Boas Práticas de Governança Corporativa para Organizações de Capital Fechado. 2014. Disponível em: http://www.ibgc.org.br/userfiles/files/AudPub.pdf?_akacao $=1793926 \&$ akcnt=98ff2d07\&_akvkey=9c99\&utm_source=akna\& utm medium=email\&utm campaign=|BGC\%3A+Audi\%EAncia+P\%FAblica+-

+Caderno+de+Governan\%E7a+para+Empresa+de+Capital+Fechado. Acessado em: 17.12.2015.

LÖFFER, Elke. BOVAIR,A.G. Public Managemente and Governance, 2ª Ed, NUC: Routledge,2009

MAHNKE, V. PEDERSEN, T. Knowledge governance and value creation. Knowledge Flows, Governance and the Multinational Enterprise: Frontiers in International Management Research, 2003

MAHNKE, V. PEDERSEN, T. Knowledge flows, governance and the multinational enterprise: Frontiers in international management research. Knowledge Flows, Governance and the Multinational Enterprise: Frontiers in International Management Research, 2003.

MARCONI, M. A., LAKATOS, E. M. (2009). Fundamentos de metodologia científica. São Paulo: Atlas.

MAYER, K.J. Spillovers and governance: An analysis of knowledge and reputational spillovers in information technology. Academy of Management Journal, 2006.

MICHAILOVA, S. SIDOROVA, E. From group-based work to organisational learning: The role of communication forms and knowledge sharing. Knowledge Management Research and Practicem, 2011.

MICHAILOVA, S. FOSS, N.J. Knowledge Governance: Themes and Questions. Knowledge Governance: Processes and Perspectives, 2009.

MITROFF, I; MASON,R.; PEARSON,C. Framebreak : the radical redesign of American business.San Francisco: Jossey-Bass, 1994

MÜLLER, R. GLÜCKLER, J. AUBRY, M. SHAO, J. Project management knowledge flows in networks of project managers and project management offices: A case study in the pharmaceutical industry. Project Management Journal, 2013.

NADAI, F. C.; CALADO, L. R. O conhecimento como recurso estratégico: caracterizando uma organização intensiva em conhecimento (OIC). In: VIII SEMEAD-SEMINÁRIOS EM ADMINISTRAÇÃO, 2005. São Paulo. Anais... São Paulo: FEA-USP, 2005

NONAKA, I.; TAKEUCHI, H. Criação de conhecimento na empresa. 5ª . ed. Rio de Janeiro: Campus, 1997.

NONAKA, I.; TOYAMA R.; HIRATA, T. Managing flow: a process theory of the knowledge-based firm. New York: Palgrave MacMillan, 2008

NOOTEBOOM, B. Learning by interaction: Absorptive capacity, cognitive distance and governance. Journal of Management and Governance 4: 69-92, 2000.

OCDE (2016), Princípios de Governo das Sociedades do G20 e da OCDE, Éditions OCDE, Paris. http://dx.doi.org/10.1787/9789264259195-pt

PAI, L.D. GOVERNANÇA CORPORATIVA \& ÉTICA NAS ORGANIZAÇÕES. Saber Acadêmico. Revista Multidisciplinar da Uniesp São Paulo: Uniesp - $\mathrm{n} \cong 06$ - Dez. 2008

PELTOKORPI, V., TSUYUKI, E. Organizational governance in internal hybrids: A case study of Maekawa Manufacturing Ltd. Corporate Governance, 2007.

PEMSEL, S. MÜLLER, R. SÖDERLUND, J. Knowledge Governance Strategies in Project-based Organizations. Long Range Planning, 2016.

PEMSEL, S. MÜLLER, R. The governance of knowledge in project-based organizations. International Journal of Project Management, 2012. 
PEMSEL, S. WIEWIORA, A. MÜLLER, R. AUBRY, M. BROWN, K. A conceptualization of knowledge governance in projectbased organizations. International Journal of Project Management, 2014.

RITTA, C. O.; ENSSLIN, S. R. Investigação sobre a relação entre ativos intangíveis e variáveis financeiras: um estudo nas organizações brasileiras pertencentes ao Índice IBovespa nos anos de 2007 e 2008. In: 10ํCONGRESSO USP DE CONTROLADORIA E CONTABILIDADE, 2010. Anais... São Paulo, 2010.

SANTISO. International co-operation for democracy and good governance: Moving towards a second generation? European Journal of Development Research, 2001.

SVEIBY, K. E. A nova riqueza das organizações: gerenciando e avaliando patrimônios de conhecimento. Rio de Janeiro: Campus, 1998.

VALE, M. CALDEIRA, J. Fashion and the governance of knowledge in a traditional industry: The case of the footwear sectoral innovation system in the northern region of portugal. Economics of Innovation and New Technology, 2008.

VALE, M. CALDEIRA, J. Proximity and knowledge governance in localized production systems: The footwear industry in the north region of Portugal. European Planning Studies, 2007.

VALE, M. Innovation and knowledge driven by a focal corporation: The case of the Autoeuropa supply chain. European Urban and Regional Studies, 2004.

VAN BUUREN, A. Knowledge for governance, governance of knowledge: Inclusive knowledge management in collaborative governance processes. International Public Management Journal, 2009.

WORLD BANK. (1992), Governance and development. Washington, Oxford University Press.

ZYNGIER, S. Governance of knowledge management. Encyclopedia of Knowledge Management, 2010.

ZYNGIER, S. BURSTEIN, F. MCKAY, J. Governance of strategies to manage organizational knowledge: A mechanism to oversee knowledge needs. Case Studies in Knowledge Management, 2005. 


\section{Dados dos autores}

Patricia de Sá Freire

Professora Doutora no Programa de Pós-graduação em Engenharia e Gestão do Conhecimento da Universidade Federal de Santa Catarina (PPGEGC/UFSC).

patriciadesafreire@gmail.com

\section{Gertrudes Aparecida Dandolini}

Professora Doutora no Programa de Pós-graduação em Engenharia e Gestão do Conhecimento da Universidade Federal de Santa Catarina (PPGEGC/UFSC).

gertrudes.dandolini@ufsc.br

\section{João Artur de Souza}

Professor Doutor no Programa de Pós-graduação em Engenharia e Gestão do Conhecimento da Universidade Federal de Santa Catarina (PPGEGC/UFSC).

jartur@gmail.com

\section{Talita Caetano Silva}

Doutoranda no Programa de Pós-graduação em Engenharia e Gestão do Conhecimento da Universidade Federal de Santa Catarina (PPGEGC/UFSC).

tcsilvaa@gmail.com

\section{Rogéria Moreira Couto}

Mestranda no Programa de Pós-graduação em Engenharia e Gestão do Conhecimento da Universidade Federal de Santa Catarina (PPGEGC/UFSC).

rogeriacouto@hotmail.com

Recebido - Received: 2017-05-19

Aceitado - Accepted: 2017-12-31

\section{(cc) EY}

This work is licensed under a Creative Commons Attribution 4.0

United States License.

\section{ULIS D-Sonk}

This journal is published by the University Library System of the University of Pittsburgh as part of its D-Scribe Digital Publishing Program and is cosponsored by the University of Pittsburgh Press. 